\title{
Jasmonate-induced plant defenses and prey availability impact the preference and performance of an omnivorous stink bug, Podisus maculiventris
}

\author{
Jennifer S. Thaler • Elena L. Olsen • \\ Ian Kaplan
}

\begin{abstract}
Many omnivorous insects are important biological control agents, and their success is influenced by both plant resistance and prey availability. The potential impact of these two factors is complicated by the fact that they may not be independent: Resistant plants also often support fewer herbivorous prey. We studied how lifetime development, growth, fecundity and preference of the omnivorous stink bug, Podisus maculiventris, was affected by jasmonate-induced plant defenses and amount of prey available. P. maculiventris survival was $70 \%$ lower on high-resistance (jasmonate-overexpressing) plants compared to low-resistance (jasmonate-insensitive) plants. However, surviving $P$. maculiventris grew and achieved equal fecundity on low- and high-resistance plants. When given a choice, $P$. maculiventris preferred low-resistance plants, but did not differentiate between caterpillar prey reared on high- or low-resistance plants. Low prey availability impacted distinct aspects of $P$. maculiventris performance: Development time was lengthened, and nymphal
\end{abstract}

J. S. Thaler $(\bowtie) \cdot$ E. L. Olsen

Department of Entomology, Cornell University, 4138 Comstock Hall, Ithaca, NY 14853, USA

e-mail: jst37@cornell.edu

\section{J. S. Thaler}

Department of Ecology and Evolutionary Biology, Cornell University, Ithaca, NY 14853, USA

\section{E. L. Olsen}

Department of Biology, University of Fribourg, 1700 Fribourg, Switzerland

I. Kaplan

Department of Entomology, Purdue University, West Lafayette, IN 47907, USA and adult mass were reduced, but survival was not impacted. We did not detect any interactive effects between plant resistance and prey availability for any measure of $P$. maculiventris. Thus, we found remarkably compartmentalized impacts of plant resistance and prey availability for this omnivorous insect.

Keywords Host plant resistance - Omnivory · Plant-insect interactions $\cdot$ Tomato $\cdot$ Heteroptera

\section{Introduction}

Omnivores are widely distributed in natural landscapes and employed in biological control, and their biology suggests that their success as predators may be particularly susceptible to plant quality (Coll and Guershon 2002; Eubanks and Denno 1999; Zhi et al. 2006). The predatory activity of omnivores may be impacted directly by host plant resistance through contact or feeding and indirectly via a reduction in the number and quality of herbivorous prey. In addition, prey scarcity may lead to increased plant feeding which could be detrimental on resistant plants (Eubanks and Denno 1999). Although strict predators may also be susceptible to these factors, omnivores are predicted to have more exposure to plant defenses because of their plant feeding and therefore be more strongly affected by these defenses. There is a conflict for successful biological control using omnivores-low-resistance plants frequently attract, provide abundant prey food, and promote survival and population growth of omnivores; however, on lowresistance plants, omnivores may feed more on plants and less on prey (Agrawal et al. 1999).

A recent meta-analysis of the effects of host plant resistance on heteropteran omnivores found that omnivore 
abundance in the field is frequently lower on resistant plants (32\% of cases), but performance does not differ (Kaplan and Thaler 2011). Nonetheless, few studies have examined how induced plant defenses influence omnivore performance and how these responses compare to and interact with prey availability. Because herbivorous prey are often less abundance on poor-quality plants, resistant plants and low prey availability are stresses that will likely be encountered simultaneously. There are multiple attributes of performance, and they may be differentially affected by plant quality and prey availability. We studied plant-induced defenses because these defenses are deployed when herbivores feed on the plant and thus when prey feeding by omnivores could benefit the plant.

The jasmonic acid pathway is the major induced defense system in plants that protects against insect attack. The near universal jasmonate pathway signals induced resistance to herbivores from a broad taxonomic range and among nearly all feeding guilds (Creelman and Mullet 1997; Thaler et al. 2002). Chewing herbivores such as caterpillars and beetles, phloem feeders such as aphids (Goggin 2007) and whiteflies (Walling 2000) and cell content feeders such as spider mites and thrips ( $\mathrm{Li}$ et al. 2002; Thaler et al. 2002) are typically negatively impacted by jasmonate-induced plant defenses. This diversity of effects of the jasmonate pathway is likely linked to the multiple classes of defense traits regulated. For example, in tomato plants, the jasmonate pathway induces trichomes, toxins, digestibility reducers and volatile signals (Kim et al. 2011; Tian et al. 2012). However, little research has been conducted on how jasmonate-induced resistance affects omnivore performance (Zhi et al. 2006).

A few studies have shown that induced plant responses can change omnivore preferences for plants versus prey. Agrawal and Klein (2000) showed that induced resistance in cotton plants caused omnivorous thrips to feed less on plants and more on prey. Herbivore damage also makes cucumber and tobacco plants more attractive to omnivores (Venzon et al. 1999; Kessler et al. 2004). These studies demonstrate the importance of induced plant responses in omnivore preference but do not pinpoint the role of jasmonate defenses. Understanding what kinds of defenses affect omnivores will be important in tailoring plant resistance strategies in agriculture that promote the compatibility of top-down and bottom-up effects.

Here, we investigated the influence of jasmonateinduced defenses of tomato plants and prey scarcity on the lifetime performance and egg production of the omnivorous heteropteran Podisus maculiventris (spined soldier bug). P. maculiventris consume plant sap with a piercingsucking stylet (Armer et al. 1998; Lambert 2007), and their feeding damage can induce the jasmonate pathway (Kim et al. 2011). Our previous field experiments showed that rates of predation by $P$. maculiventris on Manduca sexta caterpillars were lower on plants overexpressing the jasmonate pathway compared to plants not expressing the JA pathway (Kaplan and Thaler 2010), indicating that $P$. maculiventris are adversely affected by jasmonate-induced defenses. In this study, we tested the direct (through the plant) and indirect (through changes in the prey) effects of the jasmonate pathway on $P$. maculiventris performance and preference. Specifically, using tomato lines genetically altered in their expression of the jasmonate pathway, we: (1) measured the survival, growth and development time of $P$. maculiventris reared on plants with low, medium and high levels of expression of the jasmonate pathway, (2) tested whether the influence of the jasmonate pathway was altered by the level of prey availability, (3) measured whether expression of the jasmonate pathway influences host plant preference of $P$. maculiventris adults and (4) tested whether $P$. maculiventris adults had a preference for $M$. sexta caterpillars reared on plants expressing low or high levels of the jasmonate pathway.

\section{Materials and methods}

\section{Study organisms}

Podisus maculiventris were reared in a lab colony originally collected from the forest edge in Ithaca, NY using aggregation pheromone-baited traps. They were maintained on a diet of mealworms and tomato plants. The level of plant resistance via the jasmonate pathway was manipulated using three types of tomato plants that vary in the inducibility of the jasmonate pathway. Wild-type tomato plants (Solanum lycopersicon cv Castlemart) that induce the jasmonate pathway following wounding, jasmonateinsensitive mutant plants [jai-1; (Li et al. 2004)] that do not induce the JA pathway even when damaged and jasmonateoverexpressing 35S::prosystemin plants that express the jasmonic acid pathway constitutively (McGurl et al. 1994). The jasmonate-insensitive plant is a mutant defective in jasmonate perception that was backcrossed two times into the Castlemart variety, and the jasmonate overexpressor is a transgenic plant developed using agrobacterium-mediated transformation and backcrossed into the Castlemart variety. These lines vary in expression of many plant resistance traits. Jasmonate-overexpressing plants have the highest levels of defensive proteinase inhibitors and polyphenol oxidase (Howe and Ryan 1999), wild-type plants are intermediate, and jasmonate-insensitive plants have the lowest levels (Kaplan and Thaler 2010). Jasmonate-insensitive plants have $87 \%$ fewer total trichomes than wildtype or jasmonate-overexpressing plants and are absent of both the hair-like glandular type-1 trichome and the 
glandular type-VI, (Kaplan and Thaler 2010; Li et al. 2004). Therefore, jasmonate insensitive are the highest quality for many herbivores that are the prey for $P$. maculiventris, wild-type are intermediate and jasmonate overexpressor are the lowest quality (Kaplan and Thaler 2010; Rodriguez-Saona et al. 2010; Scott et al. 2010). Seeds were originally obtained from Gregg Howe (Michigan State University) and propagated by the authors in Ithaca, NY.

Seeds of the three plant types were germinated in plastic trays lined with damp paper towels and covered with plastic wrap. Jasmonate-insensitive homozygous seeds were identified with a diluted methyl jasmonate and ethanol mixture, added just after germination (Li et al. 2004). Seedlings of the three plant types were planted in metro mix soil in 4-inch pots a week after germination. Plants were grown in a climate-controlled growth chamber kept at (26 ${ }^{\circ} \mathrm{C}, 14: 10$ light: dark, 450 microeinsteins of light) until the sixth leaf stage. Plants received water regularly and fertilizer once a week (21:5:20 N:P:K, 150 ppm N). Plants grown in a growth chamber may have lower levels of defense than field grown plants, but the relative rankings of resistance in the three plant types will not be different.

How do host plant resistance and prey availability influence survival, growth and fecundity of P. maculiventris?

We manipulated host plant resistance and prey availability in a factorial design and measured the effects on $P$. maculiventris nymphal survival, growth and development. $P$. maculiventris nymphs were reared from hatching on one of six treatments: jasmonate-insensitive (hereafter, "lowresistance"), wild-type, or jasmonate-overexpressor (hereafter, "high-resistance") tomato plants with either high or low prey availability. All experiments were started with $P$. maculiventris nymphs within $24 \mathrm{~h}$ of hatching that were weighed and placed individually into 9-cm-diameter petri dishes with an excised leaflet from the appropriate plant type. Fully expanded leaflets from leaves 4, 5 or 6 were used. Fresh leaflets were exchanged every other day, and the dishes sealed with Parafilm. All nymphs were fed one mealworm $(35-60 \mathrm{mg}$ ) the day they molted into second instar (first instars do not feed on prey). Nymphs in the high prey availability treatment were given a fresh mealworm every other day; however, if the previous mealworm had not yet been eaten, they were not provided with additional food. Individuals in the low prey treatment were fed 8 days after their last kill. These starvation intervals ( 2 and 8 days) correspond with lower and upper estimates, respectively, for the length of time between prey meals for wild $P$. maculiventris foraging in the field (Legaspi et al. 1996). Nymphs were observed daily between the hours of 10 am and $1 \mathrm{pm}$, and the date of each molt and any deaths were recorded. The experiment was set up in two trials; one in July 2009 (total $n=119 ; n$ for each treatment $=13-26$ ) and one in September 2009 (total $n=120 ; n$ for each treatment $=14-24)$. Within each trial, newly hatched individuals were set up over a 2-week period with nymphs equally allocated on each day to plant type and prey availability treatments. Individuals from trial 2 were weighed on the first day of the third and fifth instars. $P$. maculiventris were kept in the study, and the plant resistance and prey availability treatments were maintained until the individual died. Adult mass was measured 3 days after adult eclosion in both trials. The number of days from egg hatch to eclosion was measured as nymphal development time. Trial 1 ran for a total of 86 days from hatching, and trial 2 ran for a total of 71 days at which point $94 \%$ of the individuals had died.

To test whether nymphal rearing conditions carried over to affect adult performance, the 35 nymphs that survived to adulthood in the second trial were used to assess fecundity. One week after adult emergence, a male was randomly selected from the colony and added to each petri dish with a female from the experiment. The adults were maintained on low-resistance plants with high prey availability. Dead males were replaced with a new individual from the colony. Petri dishes were checked each day for egg masses. Egg masses were put into individual petri dishes on a moistened filter paper. The number of eggs, date they were laid, the number that hatched, and hatch date were recorded. The day of death was recorded to determine adult lifespan.

We used Cox proportional hazards regression model (Cox and Oakes 1984) to assess how plant resistance and prey availability affected nymphal survival. We present the main effects of treatments and their interactions and coefficients for survival models as hazard ratios (exp[bi]) and standard errors. We used MANOVA to analyze third instar, fifth instar and adult mass in trial 2. When the overall MANOVA was significant, we conducted two-way ANOVAs testing the effects of plant resistance and prey availability on the individual time points. Because we had adult mass and nymphal development time for both trial 1 and trial 2, following MANOVA, these responses were analyzed using three-way ANOVA including trial. Two-way ANOVA was used to analyze adult longevity and fecundity in trial 2.

\section{Plant choice assay}

Since plant resistance affected performance of nymphs (see "Results"), choice assays were conducted to determine the preference of adult $P$. maculiventris for low and highresistance plants. Cages (24 in 3 cages, Bugdorm 2, BioQuip, Inc) were established with one low-resistance plant and one high-resistance plant set side by side approximately $30 \mathrm{~cm}$ apart. Tomato plants at the 7-10 leaf stage in 
four-inch pots were paired with plants of the other plant type based on similar size and shape. Three freshly killed mealworms (head crushed) were placed on each plant $(P$. maculiventris eat recently dead prey). One $P$. maculiventris adult was selected randomly from the colony, their sex was recorded, and placed on the floor of the cage, directly between the two pots. The position (on or off of the plant) and behavior (resting, moving, plant feeding) of the $P$. maculiventris adult was recorded $1 \mathrm{~min}$ after being placed in the cage and every 10 minutes for $1.5-2 \mathrm{~h}$. Between three and five cages were watched at a time in nine observation periods over 2 months from February to April 2010. Forty-six predators were observed.

We measured several components of plant choice: the amount of time on each plant type, the number plant feeding events on each plant type and the amount of time spent moving and resting while on a plant. Mealworm prey were rarely eaten so this behavior was not included in the analysis. We analyzed the plant choice and plant feeding data using a Pearson's Chi-square test with the null hypotheses that $P$. maculiventris would choose and plant feed equally between the low- and high-resistance plants. Signed-rank test was used to analyze the proportion of observations moving and resting.

Prey choice assay

Host plant resistance can also change the quality of herbivorous prey, so we tested whether the $P$. maculiventris adults had a preference for $M$. sexta caterpillars depending on whether caterpillars had been reared on low-resistance or high-resistance plants. $M$. sexta were grown for 6 days on each plant type when second instar individuals were matched for mass to the nearest $4 \mathrm{mg}$ and offered to adult $P$. maculiventris in 90-cm petri dishes. M. sexta caterpillars were labeled with a dot of paint on the horn, switching the color used for each treatment between replicates, so we could identify the source of the caterpillar. The $P$. maculiventris were withheld prey for $24 \mathrm{~h}$ prior to experimentation to ensure hunger. Each dish was continuously observed for $20 \mathrm{~min}$, and the first caterpillar attacked was recorded (one $P$. maculiventris did not eat a caterpillar and was excluded from the analysis; none ate more than one caterpillar). Twenty-four replicates were conducted, and the paired choices were analyzed using a sign test.

\section{Results}

Performance on high- and low-resistance plants

Survival until adulthood was strongly affected by plant resistance but not affected by prey availability. Survival was greatest on low-resistance plants where it took more than 20 days for $25 \%$ of the nymphs to die, compared to wild-type and high-resistance plants where $25 \%$ of the nymphs died within 4.5 days (Fig. 1; Table 1). On lowresistance plants, $55 \%$ of nymphs successfully reached adulthood compared to only 20 and $14 \%$, respectively, on the wild-type and high-resistance plants (Fig. 1). There was no interaction between plant resistance and prey availability (Table 1).

$P$. maculiventris mass was not affected by the level of plant resistance (Fig. 2; overall MANOVA plant resistance: $P=0.27$; Table 2); in contrast, low prey availability decreased mass (overall MANOVA $P=0.03$; Table 2). Mass was not different between nymphs in the low and high prey availability treatment when they entered the third instar, but was lower in the low prey treatment in the fifth instar and in adulthood (Table 2). Similar to mass, nymphal development time was not affected by plant
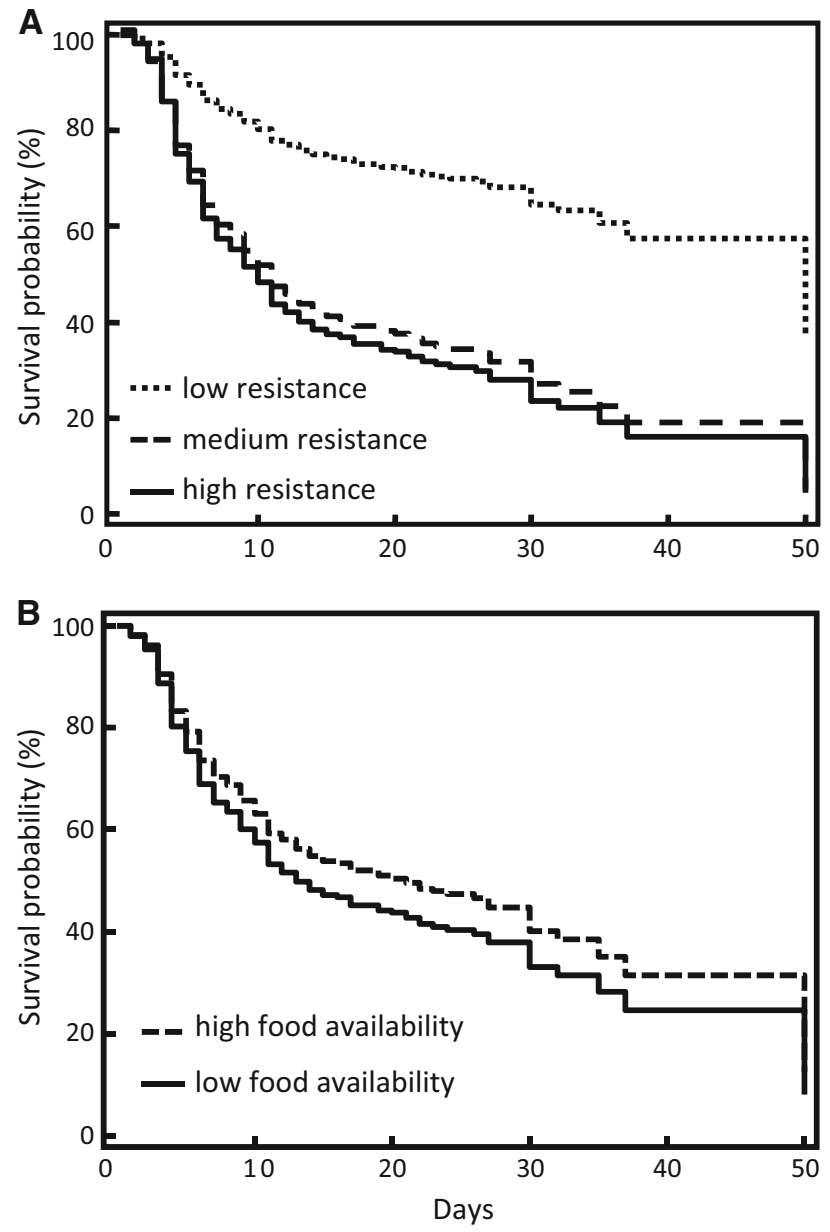

Fig. 1 The probability of nymphs surviving until adulthood. Survival was assessed daily starting when the individuals were 24-h old until they died. a On the low (jasmonate insensitive), medium (wild-type) and high-resistance (jasmonate-overexpressor) plants and $\mathbf{b}$ in the low (one mealworm every 8 days) and high (one mealworm every 2 days) prey availability treatments 
Table 1 Results from Cox proportional hazards analysis of effect of plant type and food availability on nymphal survival

\begin{tabular}{llll}
\hline & $\beta$ & $\mathrm{CI}$ & $P$ \\
\hline Plant type (wild-type) & 1.23 & $0.89-1.71$ & 0.21 \\
Plant type (low-resistance) & 0.55 & $0.37-0.81$ & 0.002 \\
Food availability (starve) & 0.76 & $0.53-1.09$ & 0.14 \\
Wild-type $\times$ starve & 1.26 & $0.79-2.011$ & 0.33 \\
Low-resistance $\times$ starve & 0.71 & $0.39-1.27$ & 0.25 \\
\hline
\end{tabular}

Plant type uses the high-resistance plant as the baseline. Food availability uses the fed treatment as the baseline. The interactions between plant type and food availability are shown as the interaction between wild-type $\times$ starve and low-resistance $\times$ starve. $\beta$ hazard ratios

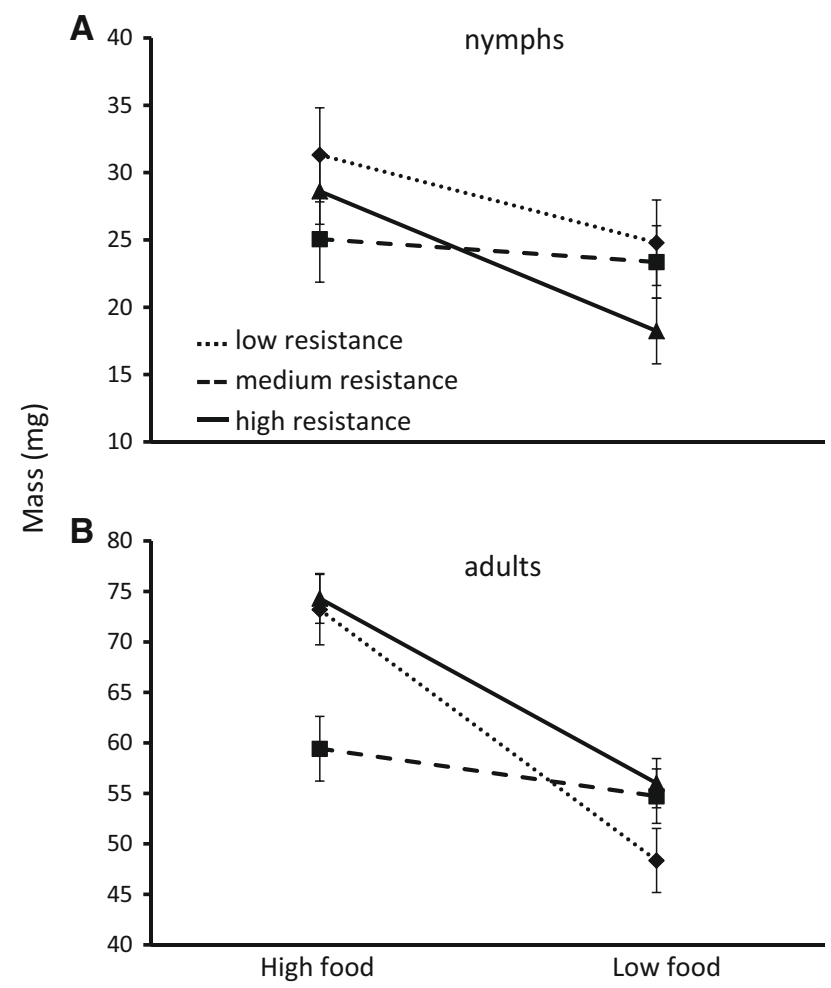

Fig. 2 The mass (mg) of P. maculiventris at a fifth instar nymphs and b 3 days after reaching adulthood in the low (one mealworm every 8 days) and high (one mealworm every 2 days) prey availability and plant resistance treatments (low $=$ jasmonate insensitive, medium $=$ wild-type high $=$ jasmonate overexpressor). Markers indicate the mean $\pm 1 \mathrm{SE}$

resistance, but was increased $23 \%$ by low prey availability (Fig. 3; Table 2). We did not detect interactions between plant resistance and prey availability on mass at any time point (Table 2).

Aside from adult mass, other components of adult performance were not affected by the plant resistance or prey availability treatments. Adult lifespan was not affected by plant resistance $(P=0.99)$, prey availability $(P=0.74)$,
Table 2 Effects of plant resistance and prey availability on mass (mg) of third and fifth instar Podisus maculiventris nymphs and adults and total nymphal development time

\begin{tabular}{|c|c|c|c|}
\hline Factor & $d f$ & $F$ & $P$ \\
\hline \multicolumn{4}{|l|}{ Mass third instar } \\
\hline Plant resistance & 2 & 0.088 & 0.96 \\
\hline Prey availability & 1 & 0.17 & 0.68 \\
\hline Plant $\times$ prey & 2 & 0.082 & 0.45 \\
\hline Error & 62 & & \\
\hline \multicolumn{4}{|l|}{ Mass fifth instar } \\
\hline Plant resistance & 2 & 0.99 & 0.38 \\
\hline Prey availability & 1 & 3.95 & 0.051 \\
\hline Plant $\times$ prey & 2 & 0.56 & 0.57 \\
\hline Error & 59 & & \\
\hline \multicolumn{4}{|l|}{ Mass Adult } \\
\hline Plant resistance & 2 & 0.34 & 0.71 \\
\hline Prey availability & 1 & 8.73 & 0.0046 \\
\hline Plant $\times$ prey & 2 & 0.46 & 0.63 \\
\hline Trial & 1 & 1.92 & 0.0080 \\
\hline Error & 54 & & \\
\hline \multicolumn{4}{|l|}{ Development time } \\
\hline Plant resistance & 2 & 1.47 & 0.23 \\
\hline Prey availability & 1 & 100.80 & $<0.001$ \\
\hline Plant x prey & 2 & 1.59 & 0.21 \\
\hline Trial & 1 & 7.41 & 0.17 \\
\hline Error & 72 & & \\
\hline
\end{tabular}

or the plant resistance $\times$ prey availability interaction $(P=$ 0.87). Males and females lived the same length of time $(P=0.38)$. Only four of the females that had been fed on the low prey diet during development reached adulthood, and one

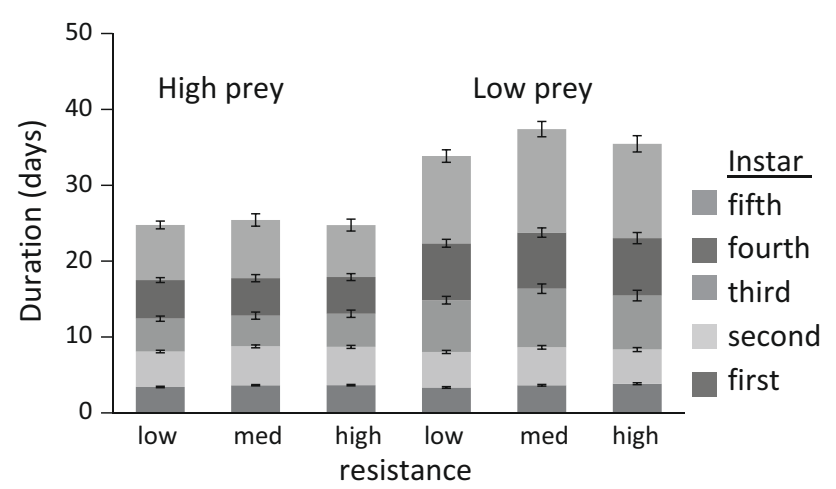

Fig. 3 The mean duration (days) of the five nymphal instars in the low (one mealworm every 8 days) and high (one mealworm every 2 days) prey availability and plant resistance treatments (low $=$ jasmonate insensitive, medium $=$ wild-type, high $=$ jasmonate overexpressor). Instar was recorded daily for the duration of the experiment. Each bar indicates the mean $\pm 1 \mathrm{SE}$ 
of these laid eggs so they were excluded from the fecundity analysis. Fourteen females laid eggs within the fed treatment, but neither egg production or number of successfully hatched eggs were affected by plant resistance (total egg production: $P=0.98$; percent egg hatch: $P=0.60$ ).

\section{Plant choice}

Because plant resistance had such a strong effect on the performance of $P$. maculiventris nymphs, we tested whether plant resistance affected adult preference. Of the $46 P$. maculiventris adults observed, 18 were found on the lowresistance plants and eight were found on the high-resistance plants (Pearson's Chi-square: $P=0.049$ ). Of the $P$. maculiventris that were observed on a plant, the average percentage of time intervals spent on the plant was $41.03 \%$ $(\mathrm{SE} \pm 6.43)$ on the low-resistance plants and $30.67 \%$ $(\mathrm{SE} \pm 11.08)$ on the high-resistance plants (Wilcoxon signed-rank test: $T=2.46, P=0.013)$. More $P$. maculiventris choose to plant feed on the low-resistance compared to the high-resistance plants. Out of 46 observed $P$. maculiventris, ten fed on the low-resistance plant and two fed on the high-resistance plant (Pearson's Chi-square: $P=0.03)$. All $P$. maculiventris plant fed only once at most and never on more than one plant. Of the $P$. maculiventris that were observed on the plants, there was no difference in the percentage of time intervals spent moving on the highand low-resistance plants $\left(F_{12}=0.077, P=0.78\right)$ or resting $\left(F_{12}=0.35, P=0.57\right)$.

Prey choice

Because plant resistance can indirectly affect omnivores through the consumption of poorer quality prey, we tested whether rearing prey on high- or low-resistance plants changed their acceptability to adult $P$. maculiventris. $P$. maculiventris did not differentiate between size-matched M. sexta caterpillars that had been reared on low-resistance (10 chosen) or high-resistance plants (13 chosen) (Sign test: $P=0.68)$.

\section{Discussion}

We report that impacts of plant resistance and prey availability are remarkably compartmentalized across the lifetime development for this omnivorous insect. Plant resistance reduced $P$. maculiventris survival, whereas low prey availability increased development time and reduced mass gain. In all nymphal instars, $P$. maculiventris survival was highest on low-resistance plants and lowest on wildtype and high-resistance plants. The higher mortality on the high-resistance plants was most pronounced in the early instars and resulted in $40 \%$ fewer reaching adulthood. However, the individuals that reached adulthood had similar performance as we did not detect an effect of plant resistance on adult mass, lifespan or fecundity. These divergent effects of the two stresses suggest that plant resistance does not affect the omnivore simply by reducing food availability, early instar nymphs are directly killed by the plant resistance traits, but do not appear to be affected by them later in life. The lack of effect of plant resistance on performance of surviving $P$. maculiventris is consistent with the results of a meta-analysis of the effects of plant resistance across many heteropteran omnivores (Kaplan and Thaler 2011).

In contrast to plant resistance, prey availability did not influence $P$. maculiventris survival, but low prey availability increased development time and reduced mass gain. Given that many polyphagous predators, including $P$. maculiventris (Legaspi and Oneil 1993, 1994a, b), are adapted to patchy food resources, maintaining survival in the face of infrequent prey meals may be the norm for this group. In a study on a closely related species, Podisus nigrispinus, Holtz et al. (2009) found that low prey availability reduced adult fecundity. Surprisingly, in our study, there were no interactions between plant quality and prey availability on omnivore performance, highlighting that plant resistance and food availability act via different routes. The independent effects of these two stresses highlight the divergent responses of omnivores to plant and prey food. These results also demonstrate the importance of measuring multiple performance traits across the omnivore's lifetime to understand how different stresses affect the organism.

The reduced survival of nymphs on resistant plants corresponds with reduced preference of $P$. maculiventris adults for high-resistance plants. As adult $P$. maculiventris did not show a preference for size-matched Manduca sexta caterpillar prey reared on high- or low-resistance plants, differences in preference appear to be driven by direct effects of plant resistance rather than indirect effects via changes in prey quality. In a study where $P$. maculiventris was fed $M$. sexta prey that had been fed on artificial diet with and without plant allelochemicals, $P$. maculiventris performance was reduced when there was both poorquality prey and prey were scarce (Weiser and Stamp 1998). While $M$. sexta are not known to sequester plant secondary compounds (Self et al. 1964; Wink and Theile 2002), they can have increased allelochemical concentrations in their hemolymph (Barbosa et al. 1991; Strohmeyer et al. 1998). This indicates that the omnivore can be affected by allelochemicals they encounter via the herbivore, and under some conditions, plant resistance and prey availability do interact to affect the omnivore.

Other studies have also found lower predation of softbodied arthropods by $P$. maculiventris on resistant plants. 
For example, a field cage no-choice experiment found that groups of $M$. sexta caterpillars on high-resistance tomato plants were eaten less than caterpillars on low-resistance plants (Kaplan and Thaler 2010). Similarly, P. maculiventris ate fewer Mexican bean beetles on resistant compared to susceptible soybean cultivars (Bartlett 2008). Since both of these studies were of short duration (several days), it suggests that $P$. maculiventris preference for lowresistance plants is what causes reduced predation on larvae.

While a large literature demonstrates the importance of the jasmonate pathway in resistance to chewing herbivores such as beetles or lepidopterans, there is less known on how the jasmonate pathway affects heteropterans, whether herbivorous or omnivorous. Several studies show that the jasmonate pathway provides resistance to herbivorous heteroptera. For instance, the mirid bug, Tupiocoris notatus, fed more on jasmonate-deficient wild tobacco plants compared to tobacco with intact defenses (Paschold et al. 2007). In contrast, cis-jasmone application to soybean plants did not affect attraction or population size of the herbivorous stink bug Euschistus heros (Vieira et al. 2013). A small number of studies have also tested the effects of the jasmonate pathway on omnivorous heteroptera. Many omnivorous heteroptera, including $P$. maculiventris (Lambert 2007), are negatively affected by trichome presence (Coll and Ridgway 1995), a trait which is regulated by the jasmonate pathway in many plant species. A specific jasmonate-regulated compound, cystatin, interferes with $P$. maculiventris midgut enzyme activity, but this did not affect their growth and development (Alvarez-Alfageme et al. 2007). Similarly, omnivorous Perillus bioculatus was not affected by a jasmonate-inducible proteinase inhibitor present in the diet of its prey (Bouchard et al. 2003).

Implications for biological control and trophic interactions

Survivorship of omnivorous $P$. maculiventris was the greatest on the same plant types where herbivory is typically greatest, plants with defective jasmonate signaling. In a scenario where $P$. maculiventris can choose between high- and low-resistance plants, this could increase biological control because the $P$. maculiventris will eat most prey on plants that are not otherwise defended by resistance traits. Our results also suggest that if older nymph move to resistant plants, their survival and performance should not be reduced. Agren et al. (2012) modeled how plant quality alters the ability of omnivores to control herbivore populations and found that the outcome is very sensitive to whether the omnivore shifts its behavior to feed on the plant or on the herbivore when on a low-quality plant. Our result suggests these omnivores would feed equally on prey coming from high- or low-quality plants but that if given a choice they would leave the low-quality plants altogether. In a mixture where high- and low-resistance plants were both available, omnivores may move to low-resistance plants which is also where the herbivores are likely most abundant.

Acknowledgments We thank Jessica McArt and Natasha Tigreros for help with survival analysis and Ordom Huot for caring for experiments over breaks. This project was supported by the National Research Initiative of the USDA Grant 2006-35302-17431 (JST), the Hunter R. Rawlings III Cornell Presidential Research Scholars program (EO) and Federal Capacity Funds 139-7484 (JST).

\section{References}

Agrawal AA, Klein CN (2000) What omnivores eat: direct effects of induced plant resistance on herbivores and indirect consequences for diet selection by omnivores. J Anim Ecol 69:525-535

Agrawal AA, Kobayashi C, Thaler JS (1999) Influence of prey availability and induced host-plant resistance on omnivory by western flower thrips. Ecology 80:518-523

Agren GI, Stenberg JA, Bjorkman C (2012) Omnivores as plant bodyguards - a model of the importance of plant quality. Basic Appl Ecol 13:441-448

Alvarez-Alfageme F, Martinez M, Pascual-Ruiz S, Castanera P, Diaz I, Ortego F (2007) Effects of potato plants expressing a barley cystatin on the predatory bug Podisus maculiventris via herbivorous prey feeding on the plant. Transgenic Res 16:1-13

Armer CA, Wiedenmann RN, Bush DR (1998) Plant feeding site selection on soybean by the facultatively phytophagous predator Orius insidiosus. Entomol Exp Appl 86:109-118

Barbosa P, Gross P, Kemper J (1991) Influence of plant allelochemicals on the tobacco hornworm and its parasitoid, Cotesia congregata. Ecology 72:1567-1575

Bartlett R (2008) Negative interactions between chemical resistance and predators affect fitness in soybeans. Ecol Entomol 33:673-678

Bouchard E, Michaud D, Cloutier C (2003) Molecular interactions between an insect predator and its herbivore prey on transgenic potato expressing a cysteine proteinase inhibitor from rice. Mol Ecol 12:2429-2437

Coll M, Guershon M (2002) Omnivory in terrestrial arthropods: mixing plant and prey diets. Annu Rev Entomol 47:267-297

Coll M, Ridgway RL (1995) Functional and numerical responses of Orius insidiosus (Heteroptera, Anthocoridae) to its prey in different vegetable crops. Ann Entomol Soc Am 88:732-738

Cox D, Oakes D (1984) Analysis of survival data. Chapman and Hall, London

Creelman RA, Mullet JE (1997) Biosynthesis and action of jasmonates in plants. Annu Rev Plant Physiol Plant Mol Biol 48:355-381

Eubanks M, Denno RF (1999) The ecological consequences of variation in plants and prey for an omnivorous insect. Ecology 80:1253-1266

Goggin FL (2007) Plant-aphid interactions: molecular and ecological perspectives. Curr Opin Plant Biol 10:399-408

Holtz AM, de Almeida GD, Fadini MAM, Zanuncio JS Jr, Zanuncio TV, Zanuncio JC (2009) Survival and reproduction of Podisus nigrispinus (Heteroptera: Pentatomidae): effects of prey scarcity and plant feeding. Chil J Agric Res 69:468-472

Howe GA, Ryan CA (1999) Suppressors of systemin signaling identify genes in the tomato wound response pathway. Genetics 153:1411-1421 
Kaplan I, Thaler JS (2010) Plant resistance attenuates the consumptive and non-consumptive impacts of predators on prey. Oikos 119:1105-1113

Kaplan I, Thaler JS (2011) Do plant defenses enhance or diminish prey suppression by omnivorous Heteroptera? Biol Control 59:53-60

Kessler A, Halitschke R, Baldwin IT (2004) Silencing the jasmonate cascade: induced plant defenses and insect populations. Science 305:665-668

Kim J, Quaghebeur H, Felton GW (2011) Reiterative and interruptive signaling in induced plant resistance to chewing insects. Phytochemistry 72:1624-1634

Lambert AM (2007) Effects of prey availability, facultative plant feeding, and plant defenses on a generalist insect predator. Arthropod Plant Interact 1:167-173

Legaspi JC, Oneil RJ (1993) Life-history of Podisus maculiventris given low numbers of Epilachna varivestis as prey. Environ Entomol 22:1192-1200

Legaspi JC, Oneil RJ (1994a) Developmental response of nymphs of Podisus maculiventris (Heteroptera, Pentatomidae) reared with low numbers of prey. Environ Entomol 23:374-380

Legaspi JC, Oneil RJ (1994b) Lipid and egg production of Podisus maculiventris (Heteroptera, Pentatomidae) under low rates of predation. Environ Entomol 23:1254-1259

Legaspi JC, Oneil RJ, Legaspi BC (1996) Trade-offs in body weights, egg loads, and fat reserves of field-collected Podisus maculiventris (Heteroptera: Pentatomidae). Environ Entomol 25:155-164

Li CY, Williams MM, Loh YT, Lee GI, Howe GA (2002) Resistance of cultivated tomato to cell content-feeding herbivores is regulated by the octadecanoid-signaling pathway. Plant Physiol 130:494-503

Li L, Zhao YF, McCaig BC, Wingerd BA, Wang JH, Whalon ME, Pichersky E, Howe GA (2004) The tomato homolog of coronatine-insensitive 1 is required for the maternal control of seed maturation, jasmonate-signaled defense responses, and glandular trichome development. Plant Cell 16:126-143

McGurl B, Orozcocardenas M, Pearce G, Ryan CA (1994) Overexpression of the prosystemin gene in transgenic tomato plants generates a systemic signal that constitutively induces proteinase-inhibitor synthesis. Proc Natl Acad Sci USA 91:9799-9802

Paschold A, Halitschke R, Baldwin IT (2007) Co(i)-ordinating defenses: naCOI1 mediates herbivore-induced resistance in
Nicotiana attenuata and reveals the role of herbivore movement in avoiding defenses. Plant J 51:79-91

Rodriguez-Saona CR, Musser RO, Vogel H, Hum-Musser SM, Thaler JS (2010) Molecular, biochemical, and organismal analyses of tomato plants simultaneously attacked by herbivores from two feeding guilds. J Chem Ecol 36:1043-1057

Scott IM, Thaler JS, Scott JG (2010) Response of a generalist herbivore Trichoplusia $n i$ to jasmonate-mediated induced defense in tomato. J Chem Ecol 36:490-499

Self LS, Hodgson E, Guthrie FE (1964) Metabolism of nicotine by tobacco-feeding insects. Nature 204:300-301

Strohmeyer HH, Stamp NE, Jarzomski CM, Bowers MD (1998) Prey species and prey diet affect growth of invertebrate predators. Ecol Entomol 23:68-79

Thaler JS, Karban R, Ullman DE, Boege K, Bostock RM (2002) Cross-talk between jasmonate and salicylate plant defense pathways: effects on several plant parasites. Oecologia 131: 227-235

Tian DL, Tooker J, Peiffer M, Chung SH, Felton GW (2012) Role of trichomes in defense against herbivores: comparison of herbivore response to woolly and hairless trichome mutants in tomato (Solanum lycopersicum). Planta 236:1053-1066

Venzon M, Janssen A, Sabelis MW (1999) Attraction of a generalist predator towards herbivore-infested plants. Entomol Exp Appl 93:305-314

Vieira CR, Blassioli Moraes MC, Borges M, Sujii ER, Laumann RA (2013) cis-Jasmone indirect action on egg parasitoids (Hymenoptera: Scelionidae) and its application in biological control of soybean stink bugs (Hemiptera: Pentatomidae). Biol Control 64:75-82

Walling LL (2000) The myriad plant responses to herbivores. J Plant Growth Regul 19:195-216

Weiser LA, Stamp NE (1998) Combined effects of allelochemicals, prey availability, and supplemental plant material on growth of a generalist insect predator. Entomol Exp Appl 87:181-189

Wink M, Theile V (2002) Alkaloid tolerance in Manduca sexta and phylogenetically related sphingids (Lepidoptera:Sphingidae). Chemoecology 12:29-46

Zhi JR, Margolies DC, Nechols JR, Boyer JE (2006) Host-plantmediated interaction between populations of a true omnivore and its herbivorous prey. Entomol Exp Appl 121:59-66 